\title{
The potential of resistant starch as a prebiotic
}

\begin{abstract}
Resistant starch is defined as the total amount of starch and the products of starch degradation that resists digestion in the small intestine. Starches that were able to resist the digestion will arrive at the colon where they will be fermented by the gut microbiota, producing a variety of products which include short chain fatty acids that can provide a range of physiological benefits. There are several factors that could affect the resistant starch content of a carbohydrate which includes the starch granule morphology, the amylose-amylopectin ratio and its association with other food component. One of the current interests on resistant starch is their potential to be used as a prebiotic, which is a non-digestible food ingredient that benefits the host by stimulating the growth or activity of one or a limited number of beneficial bacteria in the colon. A resistant starch must fulfill three criterions to be classified as a prebiotic; resistance to the upper gastrointestinal environment, fermentation by the intestinal microbiota and selective stimulation of the growth and/or activity of the beneficial bacteria. The market of prebiotic is expected to reach USD 198 million in 2014 led by the export of oligosaccharides. Realizing this, novel carbohydrates such as resistant starch from various starch sources can contribute to the advancement of the prebiotic industry.
\end{abstract}

Keyword: Functional food; Gut microbiota; Oligosaccharide; Prebiotic; Resistant starch 